\title{
„Zeit des Erwachens“ oder „Was bedeuten die konkurrierenden Risiken beim nicht kleinzelligen Bronchialkarzinom für unsere interdisziplinären Therapiekonzepte"?
}

W. Eberhardt

\author{
"Time of Awakening" or "What is the Significance of the Competing Risks in \\ Small-Cell Bronchogenic Carcinoma for our Interdisciplinary Therapy Concepts"?
}

\section{Zeit der Zufriedenheit?}

Beim lokal-fortgeschrittenen nicht kleinzelligen Bronchialkarzinom mit pulmonaler Lymphknotenmetastasierung („T3-4N1“) kann man nach den Ergebnissen der vorliegenden klinischen Untersuchung von MARRA et al. sicher nicht davon ausgehen [1]. Die Arbeit beschreibt an dem großen und ausführlich aufgearbeiteten Patientengut der Ruhrlandklinik die „ungeschminkten“ Langzeitergebnisse einer alleinigen lokalen „Therapiephilosophie“. Alle Patienten wurden primär operiert und über zwei Drittel wurden zusätzlich noch postoperativ bestrahlt. Die untersuchten 181 Patienten wurden in Essen zwischen den Jahren 1990 und 1995 diagnostiziert und behandelt. Auf der Basis der Patientencharakteristik und der Wahl der Routinebehandlung stehen diese Daten sicher durchaus repräsentativ für andere große deutsche oder auch viele internationale Therapiezentren im beschriebenen Zeitraum [2]. Dies dürfte darüber hinaus nicht nur für die Jahre 1990 bis 1995, sondern auch für die aktuelle Situation im Jahre 2002 zutreffen.

\section{Zeit der Ernüichterung!}

Die Ergebnisse der Arbeit zeigen relativ eindeutig, dass die in dieser Situation „kurativ“ geplante alleinige „Lokaltherapie“ (optimistisch bewertet) weniger als 25\% 5-Jahres-Heilungen erzielen kann. Bemerkenswert ist darüber hinaus, dass immerhin über $60 \%$ der Patienten mittels Pneumonektomie und damit ausgedehnt resezierend operiert worden sind. Als besonders ungünstig wird von den Autoren der hiläre Lymphknotenbefall („N1h“) in dieser Patientengruppe hervorgehoben und prognos- tisch dem „N2“-Befall gleichgestellt. Besonders ungünstig zeigte sich darüber hinaus auch der nachgewiesene pathologische T4-Befund (und damit das Vorliegen eines „pathologisch bestätigten IIIB-Stadiums“). Nun, wenn wir ehrlich sind, kennen wir ähnliche ernüchternde Bestandsaufnahmen schon aus anderen Untersuchungen, die die Autoren in ihrer Arbeit noch einmal sehr schön in einer ausführlichen Übersicht vergleichend gegenübergestellt haben [2-4].

\section{Zeit der Erkenntnis!}

Wir müssen die Langzeitergebnisse der hier vorgelegten Arbeit sowie die ausführlich dargestellten Rezidivmuster klar interpretieren und daraus die nötigen Konsequenzen ziehen: Wichtigste Risiken bestehen bei diesen Patienten nach der insgesamt relativ „optimierten“ Lokalbehandlung in den systemischen Rückfällen „innerhalb des Nervensystems“ (intrazerebral) bzw. „außerhalb des Nervensystems“ (sog. extrazerebrale Fernmetastasen). Die aktuellen Daten aus dem Arm mit alleiniger Lokaltherapie in der gerade vorgestellten europäischen randomisierten „ALPIEORTC“-Studie konnten übrigens diese Problematik fast parallel bestätigen: Die konkurrierenden Hauptrisiken bei den Patienten sind bei aggressiver Lokalbehandlung die extrazerebralen und erstaunlicherweise auch in diesen früheren Stadien (I - IIIA) in hohem Maße „kumulativ betrachtet“ die intrazerebralen Rückfälle [5]. 
Wie können wir nach der unzweideutigen Interpretation der vorgestellten Daten in der Essener Arbeit die Therapiekonzepte in Zukunft weiterentwickeln? Nun, wir sollten klar die erkennbaren konkurrierenden Risiken dieser Patientengruppen berücksichtigen: 1. die extrazerebralen Rezidive könnten ausschließlich durch eine systemische Therapie mit extrazerebraler Wirksamkeit beeinflusst werden. Hier bietet sich für uns momentan nur die zytotoxische (zytoreduktive) Chemotherapie als Therapieoption an. 2. da für die zytoreduktive Chemotherapie bislang keine Reduktion zerebraler Rezidivwahrscheinlichkeiten nachgewiesen werden konnte, müssen wir möglicherweise der prophylaktischen Ganzschädelbestrahlung („PCI“) auch in dieser Risikokonstellation eine neue Bedeutung zuweisen oder sie für bestimmte Subgruppen von Patienten zumindestens im Rahmen von klinischen Studien evaluieren [6,7].

Ad 1) ein wichtiges Problem besteht zum einen weiterhin in der klaren und validen präoperativen Stadieneinteilung der Patienten. Auch in der vorgelegten Untersuchung waren trotz Mediastinoskopie vor der Operation immerhin $71 \%$ der Patienten aufgrund bildgebender Verfahren den klinischen Stadien I und II zugeordnet worden. Einen möglichen Ausweg könnten hier in $\mathrm{Zu}$ kunft entweder der endobronchiale Ultraschall, die PET-(PETCT)-Untersuchung oder die MRT-Diagnostik darstellen $[8,9]$. Dieser Tatsache kommt natürlich besondere Tragweite zu, da die bislang postoperativ applizierte cisplatinhaltige Kombinationschemotherapie anscheinend nicht „effektiv zu wirken scheint" $[5,10]$.Warum dies so ist, dürfte mittlerweile besonders für die in der vorliegenden Untersuchung eingeschlossenen Patientengruppen klar belegt sein: Da der Großteil der Patienten pneumonektomiert werden muss oder alternativ komplexen Resektionsverfahren unterzogen wird, ist die Applikation einer postoperativen aggressiven Kombinationschemotherapie in höchstem Maße problematisch. Wie wir aus den meisten postoperativen Therapiestudien zur Chemotherapie nun klar wissen, liegt die postoperative Dosisintensität der Chemotherapie meist deutlich unter 50 bis $65 \%$ der eigentlich geplanten Dosierung $[5,10]$. Präoperativ lässt sich dagegen vor allem mit den neueren platinhaltigen Therapieschemen meist über $90 \%$ der geplanten Dosierung in adäquater Dosisintensität und Dosisdichte einbringen $[11,12]$.

Ad 2) Wir müssen auch für diese frühen Patientengruppen Risikokollektive identifizieren, bei denen ein besonders hohes zerebrales Rückfallrisiko existiert. Dies könnten zum Beispiel unterschiedliche Histopathologien sein: So soll das zerebrale Rückfallrisiko bei Adenokarzinomen und großzelligen Karzinomen besonders hoch sein [13]. Für diese Gruppen stellt innerhalb von intensiven, multimodalen Behandlungsprotokollen die elektive Ganzschädelbestrahlung eine durchaus zu testende Therapiekomponente dar. Sinn dürfte sie allerdings nur in Kombination mit einer systemischen Behandlung des extrazerebralen Rückfallrisikos machen.
Eine selbstkritische Analyse sollte den Weg in die Zukunft klar aufzeigen: Wir müssen in Deutschland viel mehr Patienten in diesen Risikostadien innerhalb von prospektiven Studien mittels multimodaler Behandlungsprotokolle behandeln! Optimal sollten diese Studien natürlich Vergleichsarme mit alleinigen lokaltherapeutischen Ansätzen beinhalten. Für die in der vorliegenden Untersuchung beschriebenen Patientenkollektive erscheint allerdings eine postoperative Chemotherapieapplikation innerhalb z. B. laufender Adjuvansstudien nicht unproblematisch. Die hohe Rate an letztlich doch notwendigen Pneumonektomien oder anderen komplexen Resektionsverfahren spricht eher für die präoperative Applikation einer effektiven Chemotherapie, da Compliance und Dosisintensität bei diesem Ansatz eindeutig günstiger liegen. Möglicherweise müssen wir das „Phänomen der konkurrierenden Risiken“ auch klinisch besser verstehen lernen: Vielleicht können wir die Wirksamkeit der systemischen Chemotherapie nur dann eindeutig nachweisen, wenn wir gleichzeitig das zerebrale Rückfallrisiko der Patienten mittels PCI weitgehend minimieren!? Auf jeden Fall zwingt uns diese Realität zu einer klaren, interdisziplinären Weiterentwicklung der Therapiekonzepte für unsere Patienten. Wir müssen also in Zukunft die pneumologische Risikoevaluation (pulmonale Reserven?, Comorbiditätsprofil?), die internistisch-onkologische Gesamtprognose und Therapierbarkeit (Chemotherapie? Art der Kombination? Dosisintensität?), die radiotherapeutischen Therapieoptionen ( $\mathrm{PCI}$, lokale Radiotherapie?) und die operativen Interventionen (organsparende Eingriffe?) besser in großen multizentrischen Therapiestudien optimieren. Für diejenigen, die meinen, „des Rätsels Lösung“ würde allein in der „attraktiven, modernen Immuntherapie“ liegen, muss hier allerdings deren bisherige Grenze offen aufgezeigt werden: Eine potenzielle Wirksamkeit auf a) bulky disease (z.B. wie in pulmonalen oder mediastinalen Lympknoten) oder b) zerebrale Rückfälle ist bisher für diese neue Modalität überhaupt noch nicht nachgewiesen worden [14]. Vergleichbares könnte auch auf die neuen molekular gezielten Therapien zutreffen [15]. Es könnte also sein, dass wir auch in Zukunft auf die klassischen zytoreduktiven Therapieverfahren wie Operation, Radiotherapie und Chemotherapie nicht definitiv verzichten können. Zeit also, im Sinne unserer Patienten die interdisziplinäre Therapieoptimierung zu wagen. Die Ergebnisse der letzten 10 Jahre sollten uns ja eigentlich ernüchternd klar vor Augen sein.

\section{Zeit des Mutes!}

Sicherlich können uns auch die bisherigen Ergebnisse in den eigentlichen frühen, lokalbegrenzten Stadien (IB bis IIB) nicht mit Genugtuung erfüllen! Auch hier müssen zukunftweisende, interdisziplinäre Konzepte im Rahmen von prospektiven Studien evaluiert werden, wenn wir die Heilungschancen unserer Patienten langfristig verbessern wollen. Die aktuellen Ergebnisse aus ersten Phase-II- und Phase-III-Studien zeigen übrigens, dass das alte Argument, „wir würden Patienten eine mögliche kurative Behandlungsoption vorenthalten“ eindeutig nicht stimmt. Unter einer präoperativen Induktionschemotherapie ist in den frühen, lokal begrenzten Stadien eine Progression der Erkrankung extrem selten und dürfte wohl eher auf einer schon initial vorliegenden Disseminierung beruhen $[11,12,16]$. 


\section{Literatur}

${ }^{1}$ Marra A, Hillejan L, Pöttgen $C$ et al. Bedeutung der pulmonalen Lymphknotenmetastasierung bei lokal fortgeschrittenen, nichtkleinzelligen Bronchialkarzinom. Pneumologie 2002; 56: 773 - 780

2 van Velzen E, de la Rivere AB, Elbers HJ et al. Type of lymph node involvement and survival in pathologic N1stage III non-small cell lung carcinoma. Ann Thorac Surg 1999; 67: 903 -907

${ }^{3}$ Sawyer TE, Bonner JA, Gould PM et al. Factors predicting patterns of recurrence after resection of N1 non-small cell lung carcinoma. Ann Thorac Surg 1999; 68: 1171 - 1176

${ }^{4}$ Riquet M, Manach D, Le Pimpec-Barthes F et al. Prognostic significance of surgical-pathologic N1 disease in non-small cell carcinoma of the lung. Ann Thorac Surg 1999; 67: 1572 - 1576

${ }^{5}$ Maurizio Tonato on behalf of the ALPI/EORTC-LCG investigators. Final report of the Adjuvant Lung Project Italy (ALPI): an Italian/EORTC-LCG randomised trial of adjuvant chemotherapy in completely resected non-small cell lung cancer (NSCLC). Proc Am Soc Clin Oncol 2002; 19: abstr 1157

${ }^{6}$ Cox JD, Scott CB, Byhardt RW et al. Addition of chemotherapy to radiation therapy alters failure pattern by cell type within non-small cell carcinoma of lung: analysis of Radiation Therapy Oncology Group trials. Int J Radiat Oncol Biol Phys 1999; 43: 505-509

${ }^{7}$ Stuschke M, Eberhardt W, Poettgen C et al. Prophylactic cranial irradiation in locally advanced non-small cell lung cancer after multimodality treatment: long-term follow-up and investigations of late neuropsychologic effects. J Clin Oncol 1999; 17: 2700-2709
${ }^{8}$ Herth F, Hecker E, Hoffmann H et al. Endobronchial ultrasound for local tumour and lymph node staging in patients with centrally growing lung cancer. Ultraschall Med 2002; 23: 251 - 255

${ }^{9}$ Vesselle H, Pugsley JM, Vallieres E et al. The impact of fluorodeoxyglucose $\mathrm{F} 18$ positron-emission tomography on the surgical staging of non-small cell lung cancer. J Thorac Cardiovasc Surg 2002; 124: $511-519$

${ }^{10}$ Keller SM, Adak S, Wagner $\mathrm{H}$ et al. A randomized trial of postoperative adjuvant therapy in patients with completely resected stage II or IIIA non-small-cell lung cancer. Eastern Cooperative Oncology Group. N Engl J Med 2000; 343: 1217-1222

11 Pisters KM, Ginsberg RJ, Giroux DJ et al. Induction chemotherapy before surgery for early-stage lung cancer: A novel approach. Bimodality Lung Oncology Team. J Thorac Cardiovasc Surg 2000; 119: 429-439

12 Eberhardt W, Hepp R, Korfee S et al. Current status of adjuvant and neo-adjuvant chemotherapy in resectable NSCLC. Eur Resp Rev 2002; in press

${ }^{13}$ Nussbaum ES, Djalilian HR, Cho KH et al. Brain metastases: histology, multiplicity, surgery, and survival. Cancer 1996; 78: 1781-1788

${ }^{14}$ Makita M, Hiraki A, Azuma T et al. Antilung Cancer Effect of WT1-specific Cytotoxic T Lymphocytes. Clin Cancer Res 2002; 8: 2626-2631

${ }^{15}$ Giaccone G. Clinical impact of novel treatment strategies. Oncogene 2002; 21: 6970-6981

${ }^{16}$ Depierre A, Milleron B, Moro-Sibilot D et al. Preoperative chemotherapy followed by surgery compared with primary surgery in resectable stage I (except T1N0), II, and IIIa non-small-cell lung cancer. J Clin Oncol 2002; 20: $247-253$ 